\title{
Reviewer Acknowledgements for Vol. 5, No. 2, June 2017
}

Journal of Agricultural Studies would like to acknowledge the following reviewers for their assistance with peer review of manuscripts for this issue. Many authors, regardless of whether JAS publishes their work, appreciate the helpful feedback provided by the reviewers. Their comments and suggestions were of great help to the authors in improving the quality of their papers. Each of the reviewers listed below returned at least one review for this issue

\section{Reviewers for Volume 5, Number 2}

Abhishek A. Cukkemane, Bijasu Agri Research Laboratory LLP, India

Ashit Kumar Paul, Patuakhali Science and Technology University, Bangladesh

Chenlin Hu, The Ohio State University, USA,

Eliana Mariela Werbin, National University of Cordoba, Argentinian

Ernest Baafi, CSIR-Crops Research Institute, Ghana

Gerardo Ojeda, Universidad Nacional de Colombia, Colombia

Gulzar Ahmad Nayik, SLIET Punjab, India

Jiban Shrestha, Nepal Agricultural Research Council, Nepal

Ming-Chi Wei, Department and Graduate Institute of Pharmacology, Taiwan

Reham Ibrahim Abo-Shnaf, Agricultural Research Center, Egypt

Sahar Bahmani, University of Wisconsin at Parkside, USA

Sait Engindeniz, Ege University Faculty of Agriculture, Turkey

Selmi Houc, University of Jandouba, Tunisia

Soto Caro Ariel Reinaldo, Universidad de Concepción, Chile

Zakaria Fouad Abdallah, National Research Centre, Egypt

Zhao Chen, Clemson University, US

Richard Williams,

Editor

Journal of Agricultural Studies

Macrothink Institute

5348 Vegas Dr.\#825

Las Vegas, Nevada 89108

United States

Phone: 1-702-953-1852 ext.521

Fax: 1-702-420-2900

Email: jas@macrothink.org

URL: http://jas.macrothink.org 